\title{
Hyponatraemia in the newborn
}

Hyponatraemia is common in inpatients and this includes newborns in neonatal intensive care units. Surveys from around the world suggest that up to a third of very low birthweight infants are hyponatraemic in the first week after birth and between 25 and 65\% thereafter (unpublished data). ${ }^{12}$

\section{Sodium and water balance}

How much is known about the causes of hyponatraemia in the newborn? Sodium is the principal electrolyte of extracellular fluid. The normal serum concentration in adults ranges from 132-144 $\mathrm{mmol} / \mathrm{l}$. A low serum sodium concentration reflects either an excess of water or primary sodium depletion. Water retention may occur with an increased, normal, or reduced total body sodium content. Elucidation of the cause of hyponatraemia first requires consideration of the regulation of sodium and water balance in the newborn. After birth, extracellular fluid volume contracts, and this is accompanied by net negative sodium and water balance and weight loss. ${ }^{3}$ This period is of variable duration, but by the third or fourth postnatal day, sodium and water balance have become positive, and remain so until adult life. The sodium retention that characterises growth is due to the influence of very high reninangiotensin-aldosterone system activity. The renal salt wasting seen in preterm babies below 32 weeks of gestation is due both to impaired reabsorption at the proximal tubule, resulting in a higher distal sodium delivery, and to limited aldosterone responsiveness at the distal tubule. Intestinal absorption is also limited. ${ }^{3}$

The final common pathway for the regulation of sodium balance is the $\mathrm{Na}^{+}, \mathrm{K}^{+}$ATP pump. ${ }^{4}$ In the renal tubules this is sited at the basolateral membrane. The abundance and the activity of the $\mathrm{Na}^{+}, \mathrm{K}^{+}$ATP pump increases with increasing postnatal age and are accelerated by dexamethasone treatment. Up regulation of the pump increases sodium reabsorption; down regulation increases sodium excretion. Both up regulatory and down regulatory mechanisms are immature in preterm newborns. This means that the ability both to retain and to excrete a sodium load is limited.

Both sodium and water are excreted by the kidneys, but an important potential route of additional water loss, particularly in infants born below 30 weeks of gestation, is as insensible water loss. Decreased hydrostatic and osmotic forces across the peritubular space result in decreased proximal tubular reabsorption of filtered water in newborn babies. A greater proportion of water is therefore delivered distally than in older infants. ${ }^{5}$ In the distal nephron water reabsorption is regulated by the antidiuretic hormone $(\mathrm{ADH})$ arginine vasopressin.

\section{Antidiuretic hormone}

This is present in the human posterior pituitary by the 11 th week of gestation. It has two major actions-firstly to increase the permeability of the collecting ducts by the insertion of water channels in the apical membranes of cells of the collecting ducts, ${ }^{6}$ and secondly, to cause arteriolar vasoconstriction and a rise in blood pressure. $\mathrm{ADH}$ secre- tion is triggered by alterations in tonicity of the extracellular fluid and also by baroreceptors located in the heart and great vessels. An increase in tonicity will stimulate the release of $\mathrm{ADH}$, increase the permeability of the collecting ducts, and reduce urinary water loss. The pressor effect of $\mathrm{ADH}$ seems to be one of the mediators through which central arterial blood pressure is maintained and both hypovolaemia and hypotension will cause circulating $\mathrm{ADH}$ to rise. ${ }^{7}$ Under experimental conditions, a rise in $\mathrm{ADH}$ occurs when intravascular volume falls by about $10 \%{ }^{7}$ (fig 1). Little is known about the setting of baroreceptor responses in human preterm newborns, although Rees et $a l^{8}$ described a doubling of urinary arginine vasopressin after blood loss of the order of $10 \%$, in a 26 week, $800 \mathrm{~g}$ infant.

The maintenance of central blood pressure overrides the defence of tonicity and therefore intrathoracic baroreceptor stimulation, resulting in $\mathrm{ADH}$ release; and increased water reabsorption will lead to hyponatraemia if only salt poor fluid is administered. The effect of progressive sodium depletion, with unrestricted water intake, was clearly demonstrated in 1936 by $\mathrm{McCance}^{9}$ in experiments on human volunteers. Whole body sodium depletion was initially accompanied by water loss, isotonic contraction of the extracellular compartment, and rapid weight loss. With increasing depletion, baroreceptor induced water reabsorption slowed down the rate of weight loss, but at the cost of a fall in plasma osmolality (fig 2 ).

\section{ADH secretion}

Baroreceptor stimulation of $\mathrm{ADH}$ release is often confused with the syndrome of inappropriate ADH secretion (SIADH), a term first used by Bartter and Schwartz. ${ }^{10}$ The diagnosis should be made only when circulating $\mathrm{ADH}$ is raised in the absence of both osmotic and baroreceptor mediated stimuli.

It has been suggested that the syndrome of inappropriate $\mathrm{ADH}$ secretion is common in newborns. ${ }^{8}$ Raised $\mathrm{ADH}$ activities are common in acutely ill infants. However, Gerigk et $a l,{ }^{11}$ in a large prospective study, found that although plasma osmolality was lower in acutely ill infants and children compared with a control group, both $\mathrm{ADH}$ and plasma renin activity (PRA) were raised. The greatest reduction in $\mathrm{ADH}$ and PRA activities was achieved by the intravenous infusion of isotonic saline. This suggests that the increased values were due to baroreceptor stimulation-that $\mathrm{ADH}$ was appropriately increased for volume status.

Babies born after fetal distress or after difficult delivery have higher circulating $\mathrm{ADH}$ concentrations than those born uneventfully. Pain is known to cause an immediate increase in urine concentration in babies. These effects are probably also mediated by baroreceptor stimulation.

True inappropriate $\mathrm{ADH}$ secretion is probably rare in the newborn. ${ }^{12}$ The diagnosis should be made only when hyponatraemia exists with normovolaemia, normal blood pressure, and normal renal, cardiac, adrenal and thyroid functions. There should be continuing urinary sodium loss and the urine should not be maximally dilute. ${ }^{10}$ Although 


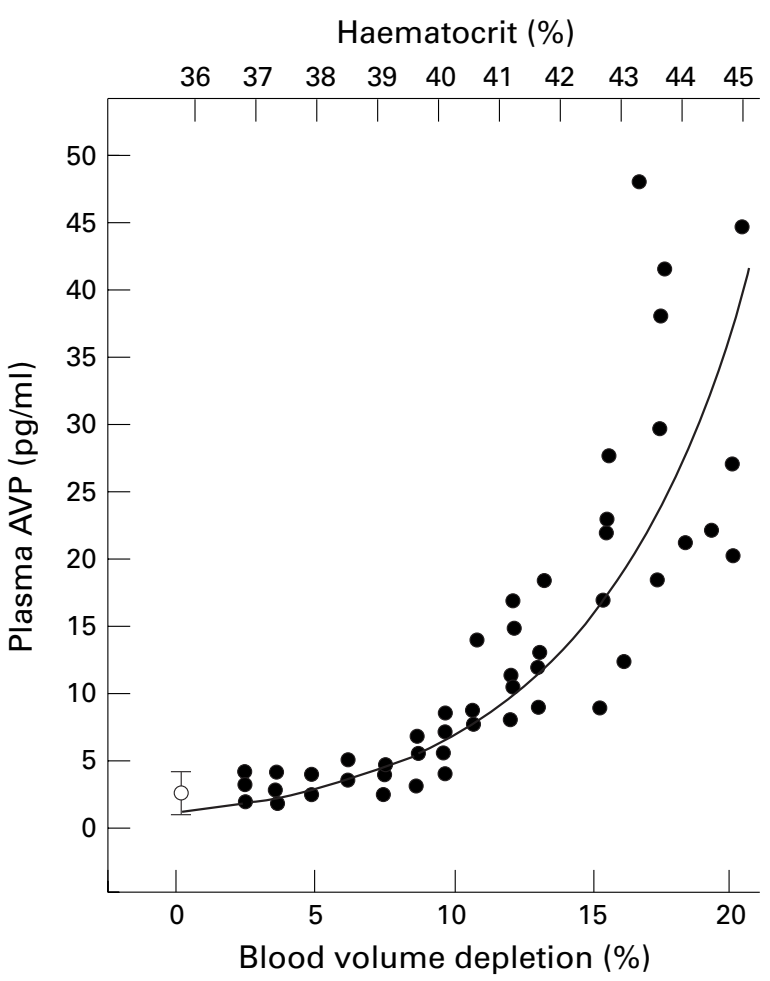

Figure 1 Relation between plasma arginine vasopressin and isosmotic changes in blood volume in rats. (Reproduced from Dunn et al) 7 Clin Invest, by copyright permission of the American Society for Clinical Investigation.

believed to occur in central nervous system infection and brain injury, reports fulfilling these diagnostic criteria are rare in neonates.

To what extent does an unrecognised reduction in central arterial blood pressure leading to baroreceptor driven reduction in free water clearance, together with the continuing provision of salt poor fluid, cause hyponatraemia during neonatal intensive care? Often, the evidence suggests. In the first place, the recognition of an inadequate intravascular volume is often difficult. In the study by Gerigk et $a l,{ }^{11}$ only a third of infants and children had overt signs of dehydration. Newborn babies are at particular risk of intravascular volume depletion. ${ }^{13}$ Immediate cord clamping can result in a $50 \%$ lower blood volume than late clamping ${ }^{14}$ and this is then compounded by frequent blood sampling. Blood pressure measurements cannot be relied on to detect hypovolaemia. The normal range for blood pressure in the newborn is wide and blood pressure correlates poorly with blood volume. ${ }^{1516}$

Impaired renal water excretion may initially be masked if transepidermal water loss is very high. Takahashi et al studied fluid balance in infants weighing less than $1000 \mathrm{~g}$, nursed in an ambient humidity above $90 \%$. Such a regimen would be expected to substantially reduce transepidermal water loss. They confirmed that mean insensible water loss was low, at less than $40 \mathrm{ml} / \mathrm{kg} / \mathrm{day}$, and remained stable throughout the first week, but $33 \%$ of the infants became hyponatraemic. Weight loss was of the order of $20 \%$, sodium supplementation began on day 3, and nutritional support consisted solely of intravenous glucose $7.5 \%$ at $50-60 \mathrm{ml} / \mathrm{kg} /$ day. This suggests that impaired free water excretion limited even greater weight loss.

Pain is often poorly controlled during neonatal intensive care and may contribute to impaired water excretion. An excessive mean airway pressure during positive pressure ventilation or high frequency oscillation will reduce cardiac output by impairing venous return to the heart. Central

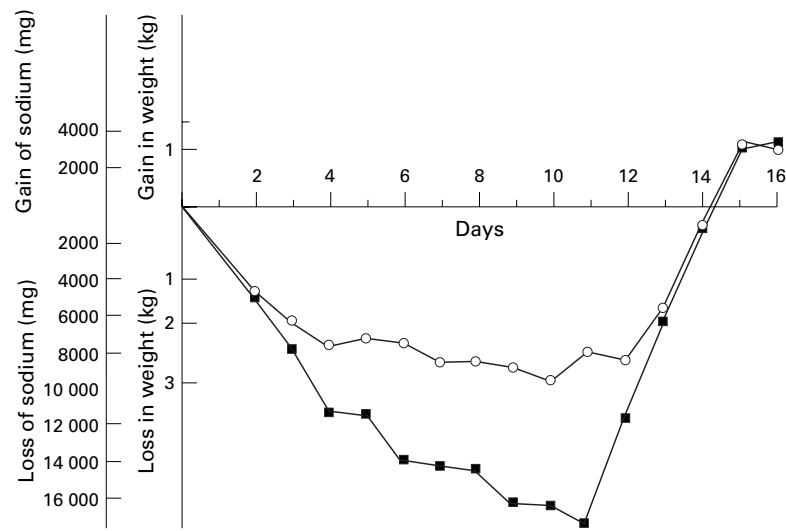

Figure 2 Changes in body sodium content and body weight during progressive salt depletion with unrestricted water intake in three human volunteers. The scales are constructed so that $1 \mathrm{~kg}$ in weight corresponds to $3300 \mathrm{mg}$ (140 mmol) sodium. (Reproduced from McCance).

venous return may also be impaired by a tension pneumothorax or other air leak syndrome.

Effective central hypotension contributes to the impaired water excretion seen in both heart failure and liver failure. In these circumstances the extracellular compartment is expanded and there may be whole body sodium excess despite the hyponatraemia. A dysfunctional myocardium, whether arising from ischaemia, metabolic acidosis, immaturity or other causes is increasingly recognised during neonatal intensive care.

\section{Chronic lung disease}

Hyponatraemia is commonly observed in infants with chronic lung disease, often with clinical signs suggestive of an expanded extracellular compartment. The aetiology of disordered salt and water balance in chronic lung disease is not adequately understood. Pulmonary hypertension, initially reversible, almost invariably accompanies chronic lung disease and fluid retention may be attributable to cor pulmonale, although cardiac performance may be impaired by other factors such as long term dexamethasone treatment.

Raised AVP has been reported in infants with chronic lung disease, together with reduced free water clearance. ${ }^{17}$. Abnormal transmural pressure gradients may lead to effective central hypotension, increased AVP release, and impaired water excretion. During acute episodes associated with air trapping, pulmonary hypovolaemia and decreased left atrial filling will lead to a similar situation. ${ }^{18}$

\section{Establishing the cause}

Hyponatraemia present at birth is a reflection of the maternal serum sodium. A large volume of salt poor fluid administered during labour will result in dilutional hyponatraemia in the neonate. Hyponatraemia present at birth has also been described following maternal diuretic and laxative abuse.

In preterm babies sodium depletion only occasionally occurs in the first days after birth, although excessive renal salt loss may occur with acute pyelonephritis or after relief of obstructive uropathy. Salt losing forms of congenital adrenal hyperplasia may also present early. In each of these cases there should be obvious evidence of extracellular volume depletion and weight loss. "Spot" urinary sodium concentrations bear no relation to daily sodium balance and are of little use in determining the cause of hyponatraemia.

After the period of postnatal extracellular volume contraction, the preterm infant is particularly vulnerable to sodium depletion as a result of immature sodium 
reabsorption. Raw breast milk and full term formulas contain inadequate quantities of sodium and the problem is compounded by the frequent use of natriuretic agents. Possible additional sources of loss are from the gastrointestinal tract and, although rare now, from repeated cerebrospinal fluid tapping. The hallmark of chronic sodium depletion is failure to gain weight appropriately.

Hyponatraemia arising suddenly is more often dilutional, due to water excess. There might be frank evidence of extracellular volume expansion and weight gain, or merely absence of anticipated weight loss. Has intake exceeded glomerular filtration capacity? Has the infant been given an excessive intravascular fluid intake? Urine volume is limited to about $20 \%$ of glomerular filtrate and infants below 28 weeks of gestation cannot increase urine output much above $8 \mathrm{ml} / \mathrm{kg} /$ hour in the first days after birth. ${ }^{5}$ In the absence of excessive insensible or gastrointestinal water loss, intravenous infusion rates in excess of $200 \mathrm{ml} / \mathrm{kg} /$ day are unlikely to be tolerated. Alternatively, is glomerular filtration reduced because of renal failure? If so, the serum creatinine will be raised, there will be anuria or oliguria, and the fractional excretion of sodium will be high.

The retention of water may be driven by $\mathrm{ADH}$. If so, the clinical challenge is to establish whether it is due to baroreceptor stimulation, or to true inappropriate ADH secretion. Careful attention should be paid to assessment of the circulation using central venous pressure monitoring, capillary refill time, and measurement of the core-peripheral temperature difference. The core-peripheral temperature difference correlates with circulating AVP. ${ }^{19}$ Is there evidence of poor myocardial function? Doppler echocardiographic indices are likely to increase in use for longitudinal assessment during neonatal intensive care. Is there evidence of impaired central venous return due to high intrathoracic pressures? Does the $x$-ray picture reveal air trapping, pneumothorax, mediastinal compression or hyperinflation? Might the infant be in pain?

The criteria for a diagnosis of SIADH are set out above. Normovolaemia and continuing urinary sodium loss will accompany hyponatraemia. The urine will not be maximally dilute. Infants below 32 weeks of gestation are able to achieve a minimum and maximum urine osmolality of around 45-90 mosm $/ \mathrm{kg}$ and $600-800 \mathrm{mosm} / \mathrm{kg}^{3}$

\section{Consequences of hyponatraemia}

Does hyponatraemia matter? The answer must be yes. Total body sodium will not only determine the size of the extracellular compartment, but sodium is also a permissive growth factor. Chronic sodium deficiency is associated with poor skeletal and tissue growth and adverse neurodevelopmental outcome. $^{20}$

Hyponatraemia and extracellular water overload increase the risks and severity of respiratory illness in the newborn, and weight gain in the first days after birth in babies with respiratory distress syndrome seems to be associated with an increased risk of developing chronic lung disease. ${ }^{3}$

It would be reasonable to assume that acute alterations in serum sodium concentrations contribute to neurological morbidity in sick neonates, although quantification of the effect, particularly in preterm babies, is difficult. Central pontine and extrapontine myelinolysis, regarded as the histopathological hallmark of hyponatraemia, has only occasionally been described in infants. ${ }^{21}$ Brain cell volume is affected by acute changes in extracellular tonicity and the regulation of cell volume following cell swelling or shrinkage in response to changes in extracellular tonicity, is brought about by the accumulation or loss of inorganic ions and organic solutes. An acute fall in serum sodium

\section{Key points}

- A low serum sodium implies absolute or relative water excess, but total body sodium may be reduced, normal or increased

- Extracellular volume contraction, weight loss, or inadequate weight gain suggests primary sodium depletion

- Extracellular volume expansion, weight gain, or failure to lose weight after birth, suggests a defect in water excretion

- Consider renal failure (oliguria, high plasma creatinine, and high fractional sodium excretion) or $\mathrm{ADH}$ driven water retention

- Both an increase in osmolality or a fall in central arterial blood pressure will stimulate the physiological release of $\mathrm{ADH}$

- A reduced effective central arterial pressure may be difficult to recognise

- The newborn in intensive care is at risk of reduced blood volume, obstructed central venous return due to high intrathoracic pressures, and poor myocardial performance

- A diagnosis of SIADH should only be made if the urine is not maximally dilute and there is continuing urinary sodium loss despite hyponatraemia, normovolaemia, and normal renal, adrenal, cardiac and thyroid function

concentration will first lead to the movement of water into cells and the development of intracellular oedema and brain swelling. Compensatory changes in electrolyte content occur rapidly, so that there is a rapid movement of electrolytes out of the cell, in favour of movement of water out of the cell. Adaptation to chronic hypo-osmolar states occurs by decreasing the concentration of intracellular organic osmolytes. ${ }^{22}$ Loss of organic osmolytes occurs more slowly than movement of electrolytes. If extracellular hypotonicity is then corrected rapidly, the continued movement of water out of the cell may result in brain shrinkage. The time scale over which the human preterm brain adapts to alterations in tonicity by increasing or decreasing intracellular organic osmolytes, is not known.

\section{Treatment}

Once acute water retention and hyponatraemia have occurred, water restriction is necessary to correct the hyponatraemia safely. In neonates severe hyponatraemia, resulting in seizures, is relatively rare, but can be controlled by the infusion of hypertonic saline. A relatively rapid rise in serum sodium in symptomatic acute hyponatraemia seems to be well tolerated in infants. ${ }^{21}$ Chronic imbalance should be corrected slowly over at least $48-72$ hours. If the water retention is caused by baroreceptor driven $\mathrm{ADH}$ secretion, the central arterial hypotension should be corrected. This may require the use of inotropic agents or the infusion of volume. It is not clear whether colloid or normal saline is to be preferred.

Chronic sodium depletion can be avoided with a sodium intake of $4-6 \mathrm{mmol} / \mathrm{kg} / \mathrm{day}$ in babies below 32 weeks of gestation, starting after the period of postnatal extracellular fluid loss, together with the replacement of additional losses. Breast milk should be fortified or a low birth weight formula used.

In infants with chronic lung disease impaired water excretion presents with inappropriate weight gain, possibly with an accompanying increase in oxygen requirement, well before obvious respiratory decompensation. A chronic 
reduction in fluid intake and therefore in nutrition, does not seem advisable, as nutritional compromise is an important concomitant of chronic lung disease. The fluid overload may be managed with diuretics such as chlorothiazide, $10-25 \mathrm{mg} / \mathrm{kg} 12$ hourly, and a potassium sparing agent such as amiloride, 200-500 $\mu \mathrm{g} / \mathrm{kg} 12$ hourly. Hyponatraemia may result from diuretic induced sodium loss but supplements should not be withheld, as chronic sodium depletion will adversely affect outcome.

\section{Future directions}

Evidence suggests that baroreceptor driven impairment in water excretion, resulting in hyponatraemia, may be common in neonates, but the issue clearly merits direct investigation. The pathophysiology of poor cardiac performance in extremely preterm infants and deranged fluid balance in chronic lung disease remains unclear. Optimal strategies for cardiovascular support during neonatal intensive care have yet to be determined.

NEENA MODI

Department of Paediatrics and Neonatal Medicine, Imperial College School of Medicine,

Hammersmith Hospital,

London W12 ONN.

1 Kloiber LL, Winn NJ, Shafffer SG, Hassanein RS. Late hyponatraemia in very low birthweight infants:incidence and associated risk factors. $\mathcal{F} \mathrm{Am}$ viet Assoc 1996;96:880-4.

2 Takahashi N, Hoshi J, Nishida H. Water balance, electrolytes and acid base balance in extremely premature infants. Acta Paediatr fpn 1994;36:250-5.

3 Modi N. Renal function, fluid and electrolyte balance. In: Roberton NRC, Modi N. Renal function, fluid and electrolyte balance. In: Roberton NRC,
Rennie JM, eds. Textbook of Neonatology. 3rd Edn. Edinburgh: Churchill Rennie JM, eds. Textbook

Livingstone. (In press.)
4 Aperia A, Holtback U, Syren ML, Svensson LB, Fryckstedt J, Greengard P. Activation/deactivation of renal $\mathrm{Na}^{+}, \mathrm{K}^{+}$-ATPase: a final common pathway for regulation of natriuresis $F A S E B \mathcal{F} 1994 ; 8: 436-9$.
5 Coulthard MG, Hey EN. Effect of varying water intake on renal function in healthy preterm babies. Arch Dis Child 1985;60:614-20

6 Deen PM, Verdijk MA, Knoers NV, et al. Requirement of human renal water channel aquaporin-2 for vasopressin dependent concentration of urine. Science 1994;264:92-5.

7 Dunn FL, Brennan TJ, Neelson AE, Robertson GL. The role of blood osmolality and volume in regulating vasopressin secretion by the rat. $\mathcal{F}$ Clin Invest 1976;52:3212-19.

8 Rees L, Brook CDG, Shaw JCL, Forsling ML. Hyponatraemia in the first week of life in preterm infants. I. Arginine vasopressin secretion. Arch Dis Child 1984;59:414-22.

9 McCance RA. Experimental sodium chloride defiency in man. Proc Roy Soc Lond (Biol) 1936;119:245-68.

10 Bartter FC, Schwartz WB. The syndrome of inappropriate secretion of antidiuretic hormone. Am F Med 1967;42:790-806.

11 Gerigk M, Gnehm HPE, Rascher W. Arginine vasopressin and renin in acutely ill children: implications for fluid therapy. Acta Paediatr 1996;85:550-3.

12 Haycock GB. The syndrome of inappropriate secretion of antidiuretic hormone. Pediatr Nephrol 1995;1 9:375-81.

13 Wardrop CA, Holland BM. The roles and vital importance of placental blood to the newborn infant. F Perinat Med 1995;23:139-43.

14 Linderkamp O, Nelle M, Kraus M, Zilow EP. The effects of early and late cord clampimg on blood viscosoty and other haemorheological parameters in full term neonates. Acta Paediatr 1992;81:745-50.

15 Barr PA, Bailey PE, Sumners J, Cassady G. Relation between arterial blood pressure and blood volume and effect of infused albumin in sick preterm infants. Pediatrics 1977;60:282-9.

16 Bauer K, Linderkamp O, Versmold HT. Systolic blood pressure and blood volume in preterm infants. Arch Dis Child 1993;69:521-2.

17 Hazinski TA, Blalock WA, Engelhardt B. Control of water balance in infants with bronchopulmonary dysplasia: role of endogenous vasopressin. Pediatr Res 1988;23:86-8.

18 Rao M, Eid N, Herrod L, Parekh A, Steiner P. Antidiuretic response in children with bronchopulmonary dysplasia during episodes of acute respiratory distress. Am F Dis Child 1986;140:825-8.

19 Lambert HJ, Coulthard MG, Palmer JM, Baylis PH, Matthews JNS. Control of sodium and water balance in the preterm neonate. Pediatr Nephrol 1990;4:C53.

20 Haycock GB. The influence of sodium on growth in infancy. Pediatr Nephrol 1993;7:871-5.

21 Gruskin AB, Sarnaik A. Hyponatraemia: pathophysiology and treatment, a pediatric perspective. Pediatr Nephrol 1992;6:280-6.

22 Strange K. Maintenance of cell volume in the central nervous system. Pediatr Nephrol 1993;7:689-97. 